\title{
Radar reconnaissance of near-Earth asteroids
}

\author{
Steven J. Ostro, Jon D. Giorgini and Lance A. M. Benner \\ Jet Propulsion Laboratory, California Institute of Technology Pasadena, California, USA \\ email: ostro@reason.jpl.nasa.gov
}

\begin{abstract}
Radar is a uniquely powerful source of information about near-Earth asteroid (NEA) physical properties and orbits. This review consists largely of edited excerpts from Ostro and Giorgini (2004).
\end{abstract}

Keywords. Minor planets, asteroids; techniques: radar astronomy

\section{Introduction}

Radar is a uniquely powerful source of information about near-Earth asteroid (NEA) physical properties and orbits. Measurements of the distribution of echo power in time delay (range) and Doppler frequency (radial velocity) constitute two-dimensional images that can provide spatial resolution finer than a decameter if the echoes are strong enough.

The best radar images reveal geologic detail, including craters and blocks. Radar wavelengths at Arecibo $(13 \mathrm{~cm})$ or Goldstone $(3.5 \mathrm{~cm})$, the world's only continuously active NEA radars (Ostro 2006a), are sensitive to bulk density (a joint function of mineralogy and porosity) and the decimeter-scale structural complexity of approximately the uppermost meter of the target's surface.

Radar can determine the masses of binary NEAs via Kepler's third law and of solitary NEAs via measurement of the Yarkovsky acceleration. With adequate orientational coverage, a sequence of images can be used to construct a three-dimensional model, to define the rotation state, to determine the distribution of radar surface properties, and to constrain the internal density distribution.

As of August 2006, radar has detected echoes from 195 NEAs, of which 110 are designated Potentially Hazardous Asteroids (Ostro 2006b). Radar has revealed both stony and metallic objects, principal-axis and non-principal-axis rotators, smooth and extremely rough surfaces, objects that appear to be monolithic fragments and objects likely to be nearly strengthless gravitational aggregates, spheroids and highly elongated shapes, contact-binary shapes, and binary systems.

Delay-Doppler positional measurements often have a fractional precision finer than 1/10,000,000, comparable to sub-milliarcsecond optical astrometry. Radar can add centuries to the interval over which close Earth approaches can accurately be predicted, significantly refining collision probability estimates compared to those based on optical astrometry alone.

If a small body is on course for a collision with Earth in this century, delay-Doppler radar could almost immediately let us recognize this by distinguishing between an impact trajectory and a near miss, and would dramatically reduce the difficulty and cost of any effort to prevent the collision. 


\section{Post-discovery astrometric follow-up}

Once an asteroid is discovered, its orbital motion must be followed well enough to permit reliable prediction and recovery at the next favorable apparition. A single radar detection of a newly discovered NEA shrinks the instantaneous positional uncertainty at the object's next close approach by orders of magnitude with respect to an optical-only orbit, thereby preventing "loss" of the object.

Comparison of radar+optical with optical-only positional predictions for recoveries of NEAs during the past decade shows that radar-based predictions have had pointing errors that average about 310 times smaller than their optical-only counterparts, dramatically facilitating recovery. Furthermore, radar astrometry (Yeomans et al. 1987; Ostro et al. 1991; see Giorgini 2006 for a tabulation) can significantly reduce ephemeris uncertainties even for an object whose optical astrometry spans many decades.

A goal of optical searches is to provide as much warning as possible of any possibly dangerous approach of NEAs as large as $140 \mathrm{~m}$. However, since an orbit estimate is based on a least-squares fit to measurements of an asteroid's position over a small portion of its orbit, knowledge of the future trajectory generally is limited by statistical uncertainties that increase with the length of time from the interval spanned by astrometry.

Trajectory uncertainties are greatest and grow most rapidly during close planetary encounters, as the steeper gravity field gradient differentially affects the volume of space centered on the nominal orbit solution within which the asteroid is statistically located. Eventually the uncertainty region grows so large, generally within the orbit plane and along the direction of motion, that the prediction becomes meaningless.

Current ground-based optical astrometric measurements typically have angular uncertainties of between 0.2 and $1.0 \operatorname{arcsec}$ (a standard deviation of 0.5 to 0.8 arcsec is common), corresponding to tens or hundreds or thousands of $\mathrm{km}$ of uncertainty for any given measurement, depending on the asteroid's distance. Radar can provide astrometry with uncertainties as small as $\simeq 10 \mathrm{~min}$ range and $\simeq 1 \mathrm{~mm} / \mathrm{s}$ in range rate. Since radar measurements are orthogonal to plane-of-sky angular measurements and have relatively high fractional precision, they offer substantial leverage on an orbit solution and normally extend NEO trajectory predictability intervals far beyond what is possible with optical data alone.

\section{Radar and collision probability prediction}

For newly discovered NEOs, a collision probability is now routinely estimated (Milani et al. 2002) for close Earth approaches, and is combined with the object's estimated diameter and the time until the approach to rate the hazard using the Palermo Technical Scale (Chesley et al. 2002). The JPL Sentry program's risk page (Chesley 2003) lists objects found to have a potential for impact within the next 100 years.

However, for newly discovered objects, the limited initial astrometry typically does not permit accurate trajectory prediction. When an object's optical astrometric arc is only days or weeks long, the orbit is so uncertain that a potentially hazardous close approach cannot be distinguished from a harmless one or even a non-existent one. The object is placed on the Sentry page, then typically removed later, when additional optical astrometry is obtained and the span of observations is extended. It is extremely rare for a radar-observed object to be on the Sentry page. 


\section{Negative predictions, positive predictions, and warning time}

To a great extent, the dominance of NEA trajectory uncertainties is a temporary artifact of the current discovery phase. Predictions are made for single-apparition objects having a few days or weeks of measurements. The uncertainty region in such cases can encompass a large portion of the inner solar system, thereby generating small but finite impact probabilities that change rapidly as the data arc lengthens, or if high-precision radar delay and Doppler measurements can be made. Impact probabilities in such cases are effectively a statement that the motion of the asteroid is so poorly known that the Earth cannot avoid passing through the asteroid's large uncertainty region - hence the apparent impact "risk". As optical measurements are made, the region shrinks. The resulting change in impact probability, up or down, is effectively a statement about where the asteroid won't be - a "negative prediction" - rather than a "positive prediction" of where it will be. This is due to the modest positional precision of optical measurements.

In contrast, radar measurements provide strong constraints on the motion and hence "positive predictions" about where an asteroid will be decades and often centuries into the future. Thus radar substantially opens the time-window of positive predictability.

\section{99942 Apophis}

The several-hundred-meter asteroid (99942) Apophis, formerly known as $2004 \mathrm{MN}_{4}$, was discovered in June 2004 and lost until it was rediscovered in December 2004. Integration of the orbit calculated from the half-year-long set of optical astrometry revealed an extremely close approach to Earth on April 13, 2029, and possibly hazardous subsequent approaches. Arecibo delay-Doppler radar astrometry obtained during late January 2005 showed the object to be several hundred kilometers closer than had been predicted by the optical measurements (Benner et al. 2005, IAU Circ. 8477). Radar observations in August 2005 (Giorgini et al. 2005, IAU Circ. 8593) and May 2006 (Benner et al. 2006, IAU Circ. 8711) further refined the orbit, moving the predicted 2036 Earth encounter to a lowerprobability region within the distribution of possible orbits. The current radar+optical collision 2036 collision probability is about one-third of the optical-only value $(\mathrm{S}$. R. Chesley, personal communication)

\section{29075 (1950 DA)}

Integrations of the radar-refined orbit of (29075) 1950 DA (Giorgini et al. 2002) revealed that in 2880 there could be a hazardous approach not indicated in the half-century arc of pre-radar optical data. During the observations, a radar time-delay measurement corrected the optical ephemeris's prediction by $7.9 \mathrm{~km}$, changing an optical-only prediction of a nominal miss distance of 20 Earth radii in 2880 into a radar-refined prediction of a 0.9-Earth-radius approach. The uncertainty in the collision probability (which could be as low as zero or as high as 1/300) is dominated by the Yarkovsky acceleration, which is due to the thermal reradiation of absorbed sunlight and depends on the object's mass, size, shape, spin state, and global distribution of optical and thermal properties. This example epitomizes the fundamental inseparability of NEA physical properties and long-term prediction of their trajectories. 


\section{Images and physical models}

With adequate orientational coverage, delay-Doppler images can be used to construct three-dimensional models (e.g., Hudson et al. 2000, 2003), to define the rotation state, and to constrain the internal density distribution. Even a single echo spectrum jointly constrains the target's size, rotation period, and sub-radar latitude. A series of Doppleronly echo spectra as a function of rotation phase can constrain the location of the center of mass with respect to a pole-on projection of the asteroid's convex envelope (e.g., Benner et al. 1999). For objects in a non-principal-axis spin state, the hypothesis of uniform internal density can be tested directly (Hudson \& Ostro 1995). Given a radar-derived model and the associated constraints on an object's internal density distribution, one can use a shape model to estimate the object's gravity field and hence its dynamical environment, as well as the distribution of gravitational slopes on the surface, which can constrain regolith depth and interior configuration.

For most NEAs, radar is the only Earth-based technique that can make images with useful spatial resolution. Therefore, although a sufficiently long, multi-apparition optical astrometric time base might provide about as much advance warning of a possibly dangerous close approach as a radar+optical data set, the only way to compensate for a lack of radar images is with a space mission.

\section{Extreme diversity}

As reviewed by Ostro et al. (2002), NEA radar has revealed both stony and metallic objects, principal-axis and complex rotators, very smooth and extraordinarily rough surfaces, objects that must be monolithic and objects that almost certainly are not, spheroids and highly elongated shapes, objects with complex topography and convex objects virtually devoid of topography. It is meaningless to talk about the physical characteristics of a "typical" NEA.

\section{Surface roughness and bulk density}

Porous, low-strength materials are very effective at absorbing energy (Asphaug et al. 1998). The apparently considerable macroporosity of many asteroids (Britt et al. 2002) has led Holsapple (2004) to claim that explosive deflection methods may be ineffective, even for a non-porous asteroid if it has a low-porosity regolith only a few cm deep.

The severity of surface roughness would be of concern to any reconnaissance mission designed to land or gather samples. The wavelengths used for NEAs at Arecibo (13 $\mathrm{cm})$ and Goldstone $(3.5 \mathrm{~cm})$, along with the observer's control of the transmitted and received polarizations, make radar experiments sensitive to the surface's bulk density and to its roughness at cm-to-m scales (e.g., Magri et al. 2001). Bulk density is a function of regolith porosity and grain density, so if an asteroid can confidently be associated with a meteorite type, then the average porosity of the surface can be estimated. Values of porosity estimated by Magri et al. (2001) for nine NEAs range from 0.28 to 0.78 , with a mean and standard deviation of $0.53+0.15$. The current results suggest that most NEAs are covered by at least several centimeters of porous regolith, so the above warning by Holsapple may be valid for virtually any object likely to threaten collision with Earth.

The fact that NEAs' circular polarization ratios (SC/OC) range from near zero to near unity means that the cm-to-m structure on these objects ranges from negligible to much more complex than any seen by the spacecraft that have landed on Eros (whose SC/OC is about 0.3, near the NEA average), the Moon, Venus, or Mars. 


\section{Binary NEAs: mass and density}

The most basic physical properties of an asteroid are its mass, its size and shape, its spin state, and whether it is one object or two (or more; Shepard et al. 2006). The current Arecibo and Goldstone systems are uniquely able to identify binary NEAs and at this writing have observed 20, most of which are designated PHAs (see the chronological history table in Ostro 2006b). Current detection statistics, including evidence from optical lightcurves (Pravec 2003) suggest that between 10\% and 20\% of PHAs are binary systems.

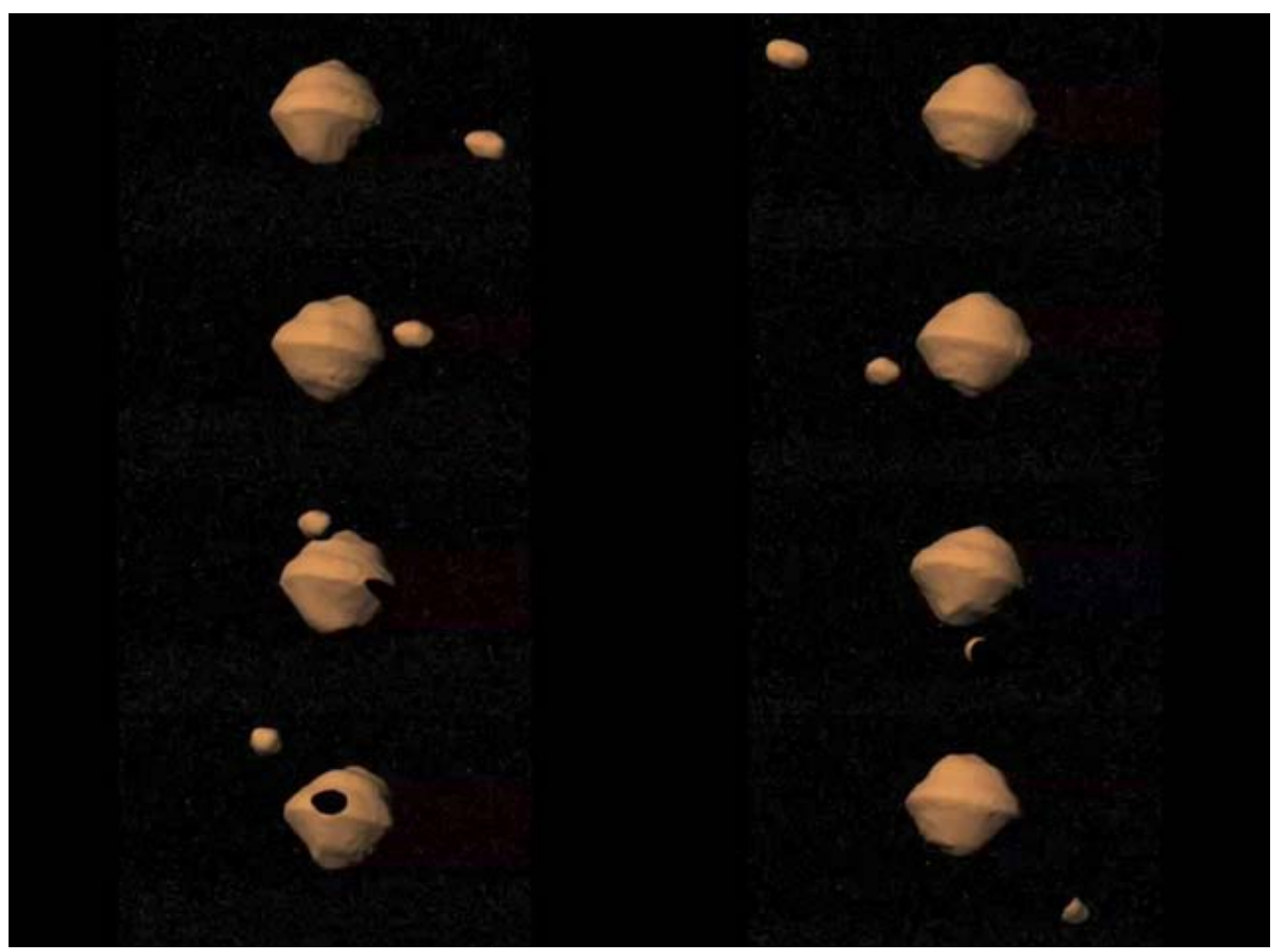

Figure 1. The asteroid (66391) $1999 \mathrm{KW}_{4}$ is a binary for which there are excellent radar data, allowing for a detailed shape model of both primary and secondary.

Analysis of echoes from these binaries is yielding our first measurements of PHA densities. Delay-Doppler images of $2000 \mathrm{DP}_{107}$ (Margot et al. 2002) reveal a 800-m primary and a 300-m secondary. The orbital period of $1.767 \mathrm{~d}$ and semimajor axis of $2620+$ $160 \mathrm{~m}$ yield a bulk density of $1.7+1.1 \mathrm{~g} \mathrm{~cm}-3$ for the primary. DP107 and other radar binaries have spheroidal primaries spinning near the breakup point for strengthless bodies. Whether binaries' components were mutually captured following a highly dispersive impact into a much larger body (Richardson et al. 2002 and references therein) or formed by tidal disruption of an object passing too close to an inner planet (Margot et al. 2002), it seems likely that most of the primaries are unconsolidated, gravitationally bound aggregates, so Holsapple's warning applies to them. 


\section{Mission design and spacecraft navigation}

Whether a PHA is single or binary, mitigation will involve spacecraft operations close to the object. Maneuvering near a small object is a nontrivial challenge, because of the weakness and complexity of the gravitational environment (Scheeres et al. 2000). Maneuvering close to either component of a binary system would be especially harrowing.

The instability of close orbits looms as such a serious unknown that unless we have detailed information about the object's shape and spin state, it would be virtually impossible to design a mission capable of autonomous navigation close to the object. Control of a spacecraft operating close to an asteroid requires knowledge of the asteroid's location, spin state, gravity field, size, shape and mass, as well as knowledge of any satellite bodies that could pose a risk to the spacecraft. Radar can provide information on all these parameters. Knowledge of the target's spin state as well as its shape (and hence nominal gravity harmonics under the assumption of uniform density; Miller et al. 1999) would permit design of stable orbits immune to escape or unintended surface impact. (Upon its arrival at (433) Eros, the NEAR Shoemaker spacecraft required almost two months to refine its estimate of the gravity field enough to ensure reliable close-approach operations.)

If it ever turns out to be necessary to have a sequence of missions beginning with physical reconnaissance and ending with a deflection, then a radar-derived physical model would speed up this process, reduce its cost, decrease complexity in the design and construction of the spacecraft, and improve the odds of successful mitigation. [Radarderived shape models of small NEAs have made it possible to explore the evolution and stability of close orbits (e.g., Scheeres et al. 1996, 1998). This radar imaging results for Itokawa (Ostro et al. 2001, 2005) were used by the Japanese Institute of Space and Astronautical Science in planning Hayabusa's encounter.] A reduced need for contingency fuel could be significant enough to allow a smaller launch vehicle for the mission. For example, the result might save $\$ 100$ million via a switch from a Titan III launch vehicle to a Titan IIS, or $\$ 200$ million for a switch from a Titan IV to a Titan III. The ability of prior radar reconnaissance to reduce mission cost, complexity and risk was embraced by the Department of Defense in their proposed Clementine II multiple-flyby mission (Hope et al. 1997), all of whose candidate targets either had already been observed with radar (Toutatis, Golevka) or were radar observable prior to encounter (1987 OA, 1989 UR).

\section{Uniqueness of radar opportunities}

How much effort should be made to make radar observations of NEAs? For newly discovered objects, it is desirable to guarantee recovery to ensure accurate prediction of close approaches at least throughout this century. Moreover, a target's discovery apparition often provides the most favorable radar opportunity for decades and hence a unique chance for physical characterization that otherwise would require a space mission. Similarly, even for NEAs that have already been detected, any opportunity offering a significant increment in echo strength and hence imaging resolution should be exploited. Binaries and non-principal-axis rotators, for which determination of dynamical and geophysical properties requires a long, preferably multi-apparition time base, should be observed extensively during any radar opportunity.

\section{Acknowledgements}

This research was conducted at the Jet Propulsion Laboratory, California Institute of Technology, under contract with the National Aeronautics and Space Administration 
(NASA). This material is based in part upon work supported by NASA under the Science Mission Directorate Research and Analysis Programs.

\section{References}

Ahrens, T.J. \& Harris, A.W. 1992, Nature 360, 429

Asphaug E., Ostro, S.J., Hudson, R.S., Scheeres, D.J. \& Benz, W. 1998, Nature 393, 437

Benner, L.A.M., Ostro, S.J., Rosema, K.D., Giorgini, J.D., Choate, D., Jurgens, R.F., Rose, R., Slade, M.A., Thomas, M.L., Winkler, R. \& Yeomans, D.K. 1999, Icarus 137, 247

Britt, D.T., Yeomans, D., Housen, K. \& Consolmagno, G. 2002 in: W.F. Bottke, A. Cellino, P. Paolicchi \& R.P. Binzel (eds.), Asteroids III (Tucson: The University of Arizona Press), p. 485

Chesley, S.R. 2003, http://neo.jpl.nasa.gov/risks

Chesley, S.R., Chodas, P.W., Milani, A., Valsecchi, G.B. \& Yeomans, D.K. 2002, Icarus 159, 423

de Pater, I., Palmer, P., Mitchell, D.L., Ostro, S.J., Yeomans, D.K. \& Snyder, L.E. 1994, Icarus 111,489

Giorgini, J.D. 2006, http://ssd.jpl.nasa.gov/radar_data.html

Giorgini, J.D., Ostro, S.J., Benner, L.A.M., Chodas, P.W., Chesley, S.R., Hudson, R.S., Nolan, M.C., Klemola, A.R., Standish, E.M., Jurgens, R.F., Rose, R., Chamberlin, A.B., Yeomans, D.K. \& Margot, J.-L. 2002, Science 296, 132

Holsapple, K.A. 2004, in: M.J.S. Belton, D.K. Yeomans \& T.H. Morgan (eds.), Mitigation of Hazardous Comets and Asteroids (Cambridge), p. 113

Hope, A.S., Kaufman, B., Dasenbrock, R. \& Bakeris, D. 1997, in: I.M. Wytrzyszczak, J.H. Lieske \& R.A. Feldman, (eds.), Dynamics and Astrometry of Natural and Artificial Celestial Bodies, Proceedings of IAU Colloquium 165 (Dordrecht: Kluwer), p. 183

Hudson, R.S. \& Ostro, S.J. 1995, Science 270, 84

Hudson, R.S., et al. 2000, Icarus 148, 37

Hudson, R.S., Ostro, S.J. \& Scheeres, D.J. 2003, Icarus 161, 348

Magri, C., Consolmagno, G.J., Ostro, S.J., Benner, L.A.M. \& Beeney, B.R. 2001, Meteoritics Planet. Sci. 36, 1697

Margot, J.L., Nolan, M.C., Benner, L.A.M., Ostro, S.J., Jurgens, R.F., Giorgini, J.D., Slade, M.A. \& Campbell, D.B. 2002, Science 296, 1445

Milani, A., Chesley, S.R., Chodas, P.W. \& Valsecchi, G.B. 2002, in: W.F. Bottke, A. Cellino, P. Paolicchi \& R.P. Binzel (eds.), Asteroids III (Tucson: The University of Arizona Press), p. 55

Miller, J.K., Antreasian., P.J., Gaskell, R.W., Giorgini, J.D., Helfrich, C.E., Owen, W.M., Jr, Williams, B.G. \& Yeomans, D.K. 1999, Amer. Astron. Soc. Paper \# 99-463, Girdwood, Alaska.

Ostro, S.J. 1997, in: J. Remo (ed.), Near-Earth Objects: The United Nations International Conference, Annals of the New York Academy of Sciences 822, p. 118

Ostro, S.J. 2006a, http://echo.jpl.nasa.gov/〜ostro/snr

Ostro S.J. 2006b, http://echo.jpl.nasa.gov/asteroids/index.html

Ostro, S.J. \& Giorgini, J.D. 2004, in: M.J.S. Belton, D.K. Yeomans \& T.H. Morgan (eds.), Mitigation of Hazardous Comets and Asteroids (Cambridge), p. 38

Ostro, S.J., Campbell, D.B., Chandler, J.F., Shapiro, I.I., Hine, A.A., Velez, R., Jurgens, R.F., Rosema, K.D., Winkler, R. \& Yeomans, D.K. 1991, Astron. J. 102, 1490

Ostro, S.J., et al. 1996, Icarus 121, 44

Ostro, S.J., et al. 2004, Meteoritics Planet. Sci. 39, 407

Ostro, S.J., et al. 2005, Meteoritics Planet. Sci. 40, 1563

Ostro, S.J., Hudson, R.S., Benner, L.A.M., Giorgini, J.D., Magri, C., Margot, J.-L. \& Nolan, M.C. 2002, in: W.F. Bottke, A. Cellino, P. Paolicchi \& R.P. Binzel (eds.), Asteroids III (Tucson: The University of Arizona Press), p. 151

Pravec, P. 2003, http://www.asu.cas.cz/ asteroid/binneas.htm

Richardson, D.C., Leinhardt, Z.M., Melosh, H.J., Bottke, W.F. Jr. \& Asphaug, E. 2002, in: W. Bottke, A. Cellino, P. Paolicchi \& R. Binzel (eds.), Asteroids III (Tucson: The University of Arizona Press), p. 501 
Scheeres, D.J., Ostro, S.J., Hudson, R.S. \& Werner, R.A. 1996, Icarus 121, 67

Scheeres, D.J., Ostro, S.J., Hudson, R.S., Suzuki, S. \& de Jong, E. 1998, Icarus 132, 53

Scheeres, D.J., Williams, B.G. \& Miller, J.K. 2000, J. Guidance, Control and Dynamics 23, 466

Shepard, M.K., Schlieder, J., Estes, B., Magri, C., Nolan, M.C., Margot, J.-L., Bus, S.J., Volquardsen, E.L., Rivkin, A., Benner, L.A.M., Giorgini, J.D., Ostro, S.J. \& Busch, M.W. 2006, Icarus 184, 198

Yeomans, D.K., Ostro, S.J. \& Chodas, P.W. 1987, Astron. J. 94, 189 